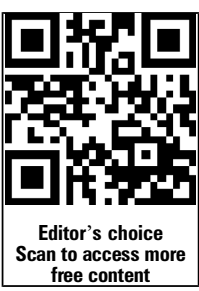
Scan to access mo
free content

\title{
Zero tolerance against patriarchal norms? A cross-sectional study of Swedish physicians' attitudes towards young females requesting virginity certificates or hymen restoration
}

\author{
Niklas Juth, Niels Lynöe
}

Stockholm Centre for Healthcare Ethics, Karolinska Institutet, Stockholm, Sweden

\section{Correspondence to} Dr Niklas Juth, Stockholm Centre for Healthcare Ethics, Karolinska Institutet, LIME, 17177 Stockholm, Sweden: niklas.juth@ki.se

Received 25 June 2013 Revised 14 January 2014 Accepted 3 February 2014 Published Online First 26 February 2014

\section{CrossMark}

To cite: Juth N, Lynöe N. $J$ Med Ethics 2015;41: 215-219.

\section{ABSTRACT}

Many countries, Sweden among them, lack professional guidelines and established procedures for responding to young females requesting virginity certificates or hymen restoration due to honour-related threats. The purpose of the present survey study was to further examine the attitudes of the Swedish healthcare professionals concerned towards young females requesting virginity certificates or hymen restorations. The study indicates that a small majority of Swedish general practitioners and gynaecologists would accommodate these patients, at least given certain circumstances. But a large minority of physicians would under no circumstances help the young females, regardless of speciality, years of practice within medicine, gender, or experience of the phenomenon. Their responses are similar to other areas where it has been claimed that society should adopt a zero tolerance policy against certain phenomena, for instance drug policy, where it has also been argued that society should never act in ways that express support for the practice in question. However, this argument is questionable. A more pragmatic approach would also allow for follow-ups and evaluation of virginity certificates and hymen restorations, as is demonstrated by the Dutch policy. Hence, there are some obvious advantages to this pragmatic approach compared to the restrictive one espoused by a large minority of Swedish physicians and Swedish policy-makers in this area.

\section{INTRODUCTION}

At a conservative estimate, some 5000 women worldwide were killed in the name of honour during $2000 .{ }^{1}$ Honour killings are triggered by allegations of extramarital sexual relations and are considered as a way of restoring the family's honour. ${ }^{2}$ Accordingly, young female $s$ or their families might try to protect themselves by requesting virginity certificates or hymen restoration from healthcare. ${ }^{2}$ One common factor explaining why young European females request these interventions seems to be their double cultural affiliation: they want to keep their affiliation to their families, often from the Middle East, and at the same time they also want to participate in the culture of their new home country. ${ }^{2-4}$ But if the young female's family suspects that she is no longer a virgin, they may require a virginity certificate. Since the idea that all young females bleed during their first intercourse is commonly embraced in honour cultures, the young female may be expected to produce red spots on the sheets during the wedding night. If a young woman has been sexually active she may thus request a hymen restoration. ${ }^{3-5}$

Different countries have developed different strategies and guidelines as to how healthcare providers should deal with patients requesting virginity certificates or hymen restorations. In Holland, two Amsterdam Hospitals, who most often deals with these problems, have developed a protocol based on a patient-centred approach. ${ }^{4}$ The point of departure in this protocol is that if the young female, after information and clinical educational examination, wants a hymen restoration operation, her request should be granted. The Dutch protocol also allows follow-ups, which have been difficult in countries where the intervention is banned or more or less tabooed. ${ }^{6}$

In other countries, for example, Sweden, there are no such guidelines. Hence, the healthcare providers concerned are uncertain how to deal with young females requesting virginity certificates or hymen restoration. ${ }^{7}$ However, the political message in Sweden is that hymen operations should be considered a non-option and that the appropriate response to such requests should be information about the medical aspects of sexuality and human rights and, if necessary, referral to police or social authorities for protection. ${ }^{8} 9$ The main argument for this practice is that Swedish society should take a stand against practices expressing control of female sexuality. The official Swedish viewpoint hence expresses a zero tolerance policy against patriarchal norms and values.

A previous survey in Sweden about healthcare staff's experience of ways of encountering such patients indicated that attitudes varied very much and that some healthcare professionals (HCP) refused to see such patients at all. ${ }^{7}$ In a recent interview study, we found that HCPs with long experience of hymen operations had to perform such operations more or less in secret. ${ }^{3}$ Since the issue in Sweden is tabooed, it is very difficult to document and evaluate the quality and effect of information, education and operations. ${ }^{3}$ The results of these Swedish studies indicate a risk of arbitrariness when encountering such patients, as regards whether or not they will be offered help. And arbitrariness is against the principle of equal access to 
healthcare for all patients, a principle embraced by the Swedish healthcare system.

The purpose of the present study was to further examine the attitudes of the Swedish HCPs concerned towards young females requesting virginity certificates or hymen restorations. The purpose was also to examine possible values underlying the physicians' attitudes.

\section{PARTICIPANTS AND METHODS}

We selected a random sample of 995 general practitioners (GPs) and 988 gynaecologists in Sweden, since physicians from these specialties are most likely to confront females requesting virginity certificates or hymen restoration. Writing a virginity certificate may be considered morally different from performing hymen restoration. ${ }^{10}$ To prevent the responses to one of the issues influencing the responses to the other, we chose to separate the two issues. We also assumed that GPs have more experience of requests for certificates and gynaecologists more experience of requests for hymen operations. Hence, two versions of the survey were distributed randomly. One included a vignette with a scenario of a young female requesting a virginity certificate. The other included a vignette with a scenario of a young female requesting a hymen restoration. Among the GPs, 499 received the certificate version and 496 the operation version. Among the gynaecologists, 494 received the certificate version and 494 the operation version.

In both scenarios, the young female expressed fear of being exposed to violence if her request was not granted. The content of all questions and response options was identical in the two versions of the questionnaire. The first question was 'The physician ought in these circumstances to perform the operation' (in the hymen version) or 'The physician ought in these circumstances to write the certificate' (in the certificate version). Response options were: agree completely, agree to a large extent, disagree to a large extent and disagree completely. The respondents were subsequently asked to state their own arguments for or against the preferred action. The next question was: 'The physician ought under no circumstances to perform the operation requested' (in the hymen version) or 'The physician ought under no circumstances to write the certificate requested'-the response options were the same as above. The respondents were also asked to state arguments of their own for and against their position in relation to this question.

We also asked the participants what would happen to their own trust in healthcare with physicians writing virginity certificates or performing hymen restorations. Response options were that trust would decrease, would not be influenced or would increase. Decreasing one's trust in healthcare due to the performed action was interpreted as equivalent to estimating the action as bad. The respondents were also asked about their experiences of young females requesting hymen operations or virginity certificates; the response options were: often, rarely and never. Finally, the respondents were asked about their sex, age, speciality and years working within the speciality (see table 1).

Since we identified no significant differences between GPs and gynaecologists, we have merged the two groups when presenting the results. Data were registered and analysed using the Epi-Info software (V.6.04d). When comparing response patterns we focused on those who disagreed completely as to under certain circumstances perform virginity certificate and hymen reconstruction and those who agreed completely with the statement as to under no circumstances perform such actions. We used $\chi^{2}$ test and also presented results as proportions with $95 \%$ CIs, assuming non-overlapping intervals to indicate that a hypothesis test might have provided a p value $<0.001$.
Table 1 Background variables presented as proportions in relation to the two specialities, sex, experience of the issue, what would happen with their trust in healthcare of virginity certificates or hymen restoration were performed regarding the two versions of the questionnaire

\begin{tabular}{lll}
\hline Questionnaire versions & $\begin{array}{l}\text { Virginity } \\
\text { certificates }\end{array}$ & $\begin{array}{l}\text { Hymen } \\
\text { restoration }\end{array}$ \\
\hline GPs ( $\mathrm{n}=443$ ) & $51 \%$ & $49 \%$ \\
Gynaecologists ( $\mathrm{n}=557)$ & $49.2 \%$ & $50.8 \%$ \\
Female GPs & $47.8 \%$ & $45.2 \%$ \\
$\quad$ Female gynaecologists & $65.3 \%$ & $67.5 \%$ \\
Median age 58 years (range 35-80) & & \\
$\quad$ GPs<58 years & $56.6 \%$ & $43.4 \%$ \\
Gynaecologists <58 years & $45.4 \%$ & $54.6 \%$ \\
Experiences of the issues & & \\
Often (GPs/gynaecologists) & $0.9 \% / 7 \%$ & $2.3 \% / 11 \%$ \\
$\quad$ Seldom (GPs/gynaecologists) & $17.3 \% / 59 \%$ & $14.9 \% / 60 \%$ \\
$\quad$ Never (GPs/gynaecologists) & $81.8 \% / 34 \%$ & $82.8 \% / 29 \%$ \\
My trust in healthcare if performed would: & \\
$\quad$ Decrease (GPs/gynaecologists) & $67 \% / 61.6 \%$ & $54.1 \% / 58.4 \%$ \\
$\quad$ Not influenced (GPs/ & $29.4 \% / 34.3 \%$ & $42.9 \% / 37.7 \%$ \\
gynaecologists) & & \\
Increase (GPs/gynaecologists) & $3.6 \% / 4.1 \%$ & $2.8 \% / 3.9 \%$ \\
\hline GPs, general practitioners. & &
\end{tabular}

The study was approved by the regional research ethics committee at Karolinska Institutet, Stockholm (DNR: 2012/ 1071-31/1).

\section{RESULTS}

Totally there were 1001 responses, resulting in an average response rate of 50.5\%. The two specialities, GPs and gynaecologists, provided almost similar attitudes to whether or not to write a virginity certificate or perform hymen restoration. On average, $46.9 \%$ (CI 41.4 to 51.4 ) of all physicians agreed completely under no circumstances to write a virginity certificate. Similar average numbers for hymen restorations were $42 \%$ (CI 37.4 to 46.6 ), indicating that a majority would be prepared to assist the young females under certain conditions. Although the GPs tended to be slightly more restrictive when writing certificates $(52.8 \%$ (CI 46 to 59.6 ) vs $42.4 \%$ (CI 36.4 to 48.4 )), the difference in restrictive attitude was not significant and almost disappeared when answers on hymen restoration were factored in. The average response rate of gynaecologists (56.4\% (CI 52.3 to 60.5$)$ ) was significantly higher compared with GPs $(44.6 \%$ (CI 40.0 to 49.2$)$ ). Although the issues were rarely experienced in general, gynaecologists seem to be significantly more familiar with both issues (see table 1). Compared with GPs, significantly more gynaecologists were females and compared with gynaecologists, GPs tended to state that their trust would be reduced more by writing virginity certificates than by hymen restoration $(p<0.05)$. Otherwise, we found no differences in attitudes towards the two issues between the two specialities regarding other background variables.

When comparing all physicians who had some experience of the issues with those who had no experience, we found that those who had experience of writing certificates tended to become less restrictive and that those who had experienced hymen operations tended to be more restrictive, but the difference was not significant. 
Table 2 Proportions (\%) of all the physicians who agree completely (AC), agree to a large extent (ALE), disagree to a large extent (DLE) or disagree completely (DC) when asked to write a virginity certificate or perform a hymen reconstruction under certain circumstances and under no circumstances and as to whether their trust would decrease or not be influenced/would be increased

\begin{tabular}{|c|c|c|c|c|c|c|c|c|}
\hline & \multicolumn{4}{|c|}{ Virginity certificate (\%) } & \multicolumn{4}{|c|}{ Hymen reconstruction (\%) } \\
\hline & $A C$ & ALE & DLE & DC & AC & ALE & DLE & DC \\
\hline \multicolumn{9}{|l|}{ Under certain circumstances } \\
\hline Trust decreases $(312 / 279)$ & 0.3 & 1.3 & 22.8 & 75.6 & 0.0 & 0.3 & 28 & 71.7 \\
\hline Trust not influenced (173/205) & 19.7 & 38.7 & 22.5 & 19.1 & 9.8 & 39.5 & 33.2 & 17.5 \\
\hline \multicolumn{9}{|l|}{ Under no circumstances } \\
\hline Trust decreases (302/266) & 64.4 & 27.5 & 4.6 & 3.3 & 63.5 & 29.7 & 4.2 & 2.6 \\
\hline Trust not influenced (160/195) & 13.1 & 23.1 & 25.6 & 38.1 & 13.8 & 28.7 & 31.8 & 25.7 \\
\hline
\end{tabular}

We identified significant differences when comparing the group of physicians whose trust would decrease with the group whose trust would not be influenced or would increase (see table 2). A majority of the group whose trust would decrease agreed completely with the statement that they would under no circumstances write a certificate or perform an operation (63.9\% (CI 59.5 to 67.9)), which can be compared with the group whose trust would not be influenced (or increased) (13.2\% (CI 9.6 to 16.8$)$ ).

\section{Open-ended questions and comments}

Arguments against hymen restorations were more frequent compared with writing certificates and included the subsequent statements about honour norms: they are dangerous, patriarchal oppressive, humiliating, religious or cultural hallucinations, fraudulent, barbarian, hypocritical, obsolete, and so on. Another common argument against helping was: 'when in Rome, do as the Romans do', that is, females in these circumstances should adapt to the majority culture rather than look for help to keep the affiliation with their traditional culture. Focusing on factual arguments for and against performing virginity certificates or hymen reconstruction, that is, arguments regarding what would actually happen if these actions were performed without explicit normative judgements, we found that opposing statements were made by different respondents depending on whether they would perform these actions under no circumstances or certain circumstances (see table 3).

\section{DISCUSSION}

Almost half of the groups of physicians studied agreed completely with the statement that they ought under no circumstances to write virginity certificates or perform hymen restorations. Yet, a small majority seems to be prepared to assist with this, given certain conditions. Otherwise, a conspicuous result was the lack of differences in response patterns between GPs and gynaecologists. First, the level of opposition against writing virginity certificates compared with performing hymen restoration is roughly quantitatively equal, even though the comments regarding hymen restoration were harsher. A previous study indicated that more experience of a course of action leads to a more tolerant attitude towards it, ${ }^{3}$ but we found no support for this assumption.

The only statistically significant difference regarding whether or not to write virginity certificates or perform hymen restorations was related to what would happen to the participants' trust in healthcare: a significantly larger number of respondents who agreed completely with the statement that they would under no circumstances write a certificate or perform an operation claimed that their trust would decrease compared with those who claimed that their trust would not be influenced or would increase. Since the scenario of jeopardising trust in healthcare must seem very undesirable to physicians in general, we might interpret this group of respondents as expressing a very strong aversion towards the two interventions. If this interpretation is correct, one can ask how this strong aversion should be understood.

\section{Zero tolerance of drugs and patriarchal norms?}

In Sweden, as well as in many other countries, similar restrictive attitudes are expressed when discussing whether or not to provide substance abusers with sterile syringe exchange. The goal of such sterile needle programmes is to reduce harm by preventing infectious diseases and complications. Those who are against such programmes find them ineffective and counterproductive. Those who are for such programmes tend to find them effective and functioning as gateways to other medical treatment. Even though the evidence seems to support the latter position, ${ }^{11} 12$ the political resistance to such programmes has been significant. The main argument against syringe exchange programmes has been that acceptance would mean society signalling or expressing that substance abuse is acceptable and, accordingly, would jeopardise the war against substance abuse. ${ }^{11}$ Instead, society should at all times express zero tolerance of drugs. $^{13}$

Table 3 Comments regarding statements that physicians should under no circumstances write virginity certificates or perform hymen reconstructions are compared with comments regarding the statement that physicians should under certain circumstances do so

\begin{tabular}{|c|c|}
\hline Under no circumstances & Under certain circumstances \\
\hline Would support patriarchal norms & It would undermine patriarchal norms \\
\hline Would jeopardise trust in healthcare & Would not influence trust in healthcare \\
\hline $\begin{array}{l}\text { Threats against the patient not } \\
\text { serious }\end{array}$ & Trust the females and take threats serious \\
\hline Illegal (against the law) & In principle against, but difficult to say no \\
\hline Not possible to certify virginity & Diplomatic certificates works \\
\hline $\begin{array}{l}\text { Hymen restoration not part of } \\
\text { healthcare }\end{array}$ & Duty to safe a patient's life \\
\hline Hymen restorations do not function & Simple way of helping patients \\
\hline $\begin{array}{l}\text { Hymen reconstruction is unsafe and } \\
\text { risky }\end{array}$ & Hymen reconstruction is safe and not risky \\
\hline
\end{tabular}


There may be an analogy between the idea of zero tolerance of drugs and zero tolerance of oppressive patriarchal norms. The most common argument among those unconditionally against virginity certificates and hymen operations was that doing these things would be to support or express patriarchal oppressive norms, an argument also expressed by Swedish politicians and authorities. ${ }^{89}$ This argument seems to be analogous to the line of reasoning against sterile syringe exchange programmes for substance abusers.

However, there are several reasons for being sceptical of this argument. First, there is no empirical evidence to suggest that healthcare professionals performing hymen operations or writing virginity certificates would increase patriarchal oppression. However, one could still maintain that these courses of action would express support for the patriarchal culture.

Against this kind of so-called expressive arguments, ${ }^{14}$ it must be noted that it is often unclear what kinds of beliefs and attitudes certain kinds of acts can be said to express. Providing hymen reconstruction and virginity certificates does not necessarily express support for the patriarchal norms that recommend them. One could actually maintain that society expresses repudiation of patriarchal norms by aiding young females to deceive its representatives. In one of the comments, it was actually stated that writing certificates or reconstructing hymens were actions that could undermine the patriarchal norms. The question is whether or not possible consequences of deceiving representatives of patriarchal norms are something we would like to happen. If the answer is yes, the goal of undermining these norms would actually support writing false certificates and performing hymen reconstruction. Also, one could say that by refusing hymen restoration and virginity certificates, society declares that it is the duty of young women, rather than society, to fight these norms, regardless of the cost to themselves.

Moreover, a zero tolerance policy makes it difficult to conduct follow-ups and accordingly to assess whether or not the interventions are effective and safe. To those gynaecologists who actually perform hymen restorations in Sweden, this is a big problem. ${ }^{3}$ Furthermore, it also becomes difficult to assess whether or not, for example, organised hymen restorations would promote a broader acceptance of old patriarchal traditions in Swedish society. Accordingly, the argument becomes irrefutable.

Evidence from Holland suggests that a patient-centred and autonomy-supporting approach makes it possible to follow-up and assess whether or not different strategies, hymen restoration included, are safe and effective. ${ }^{4}$ Furthermore, it is solely a small minority who after educational clinical examination still request hymen restoration. ${ }^{4}$ Until we can evaluate these interventions properly, the Dutch approach seems more fruitful than the restrictive one favoured in Sweden.

\section{Writing 'diplomatic' certificates}

Gynaecologists in general consider it fraudulent to write a virginity certificate on the basis of a gynaecological examination of the female genitals. ${ }^{7}$ Nevertheless, physicians sometimes do this in order to help the young female, and a certain proportion of the present study sample was actually prepared to do so under certain conditions, or at least prepared to claim that it ought to be done. Several respondents commented that it is possible to write a 'diplomatic' certificate, for example, by not explicitly stating that one found the female investigated to be a virgin but only saying that one could not establish that she was not. In that way, one could be said to avoid outright mendacity about the result of the examination. However, it was stated in the vignette of this survey that the young female was actually sexually active. By implication, it would seem that those prepared to write a virginity certificate, including a diplomatic one, are also prepared to cheat someone else by leaving out the truth, in order to help the young woman. This corroborates previous studies in which it has been found that a large proportion of physicians are willing to write false certificates when they deem it necessary to protect the life and well-being of patients, although in different circumstances. ${ }^{15}$ However, it remains difficult for physicians to openly acknowledge engaging in such deceptive practices, even if many of them are prepared to do so. ${ }^{16}$

\section{Value conflicts}

What is considered the main value conflict by responders is at one hand the importance of helping patients in distress (or saving lives) and on the other hand the importance of standing up against suppressive and patriarchal norms. Several participants who stated that they were against patriarchal norms also stated that if facing that a patient's life was at stake they were inclined to help such a patient. However, responders differ regarding their beliefs about whether writing certificates and performing hymen reconstructions would actually express or support patriarchal norms-some even claim that these actions could undermine them. We have also briefly argued that there is no necessary connection between these actions and expressing patriarchal norms. This position allows us undermining the significance of the patriarchal norms and at the same time helping the females. A similar but unarticulated attitude may also be reflected among all those (53.1\%) who would keep the door ajar by not agreeing completely to under no circumstances write a certificate or perform a hymen operation.

\section{Strengths and weaknesses}

The study had a large drop-out rate, particularly among GPs. However, we found no differences in response pattern between those who answered the first and the second reminder, so we have no independent reason to believe that a lower drop-out rate would have affected the results significantly. Although the questionnaire was conducted anonymously, it cannot be excluded that the participants responded in a way considered as politically correct: an enlightened physician is not supposed to support patriarchal norms. That might explain why so many answered that they would under no circumstances perform the interventions requested. But the response options-agreeing completely or to a large extent and disagreeing to a large extent or completely - made it possible to provide a less rigid response. Agreeing to a large extent not under any circumstances assist such a young female keeps the door ajar. But complete agreement shuts all doors. The harsh comments from the respondents who chose this alternative indicate that they expressed their gut feelings rather than their reflected opinions to a larger extent than other respondents. A study with another design, for example, interviews, might have resulted in more balanced answers.

\section{CONCLUSIONS}

The present study indicates that a majority—albeit a small one—of Swedish GPs and gynaecologists would under certain conditions help young females with virginity certificates or hymen restoration upon request. But a rather large minority of physicians would under no circumstances help the young females, regardless of speciality, years of practice within medicine, gender or experience of the phenomena. The respondents' comments suggest that the main value conflict between the majority and the minority is that the former emphasises the importance of helping patients in distress and the latter emphasises the importance of expressing opposition to suppressive and patriarchal norms. Considering the harsh comments 
from this minority and the strong association between them and those whose trust in healthcare would be jeopardised, it is likely that this group of respondents had their emotions stirred up. This is similar to other areas where it has been argued that society should adopt a zero tolerance policy against certain phenomena, for example, drug policy. A common argument for such zero tolerance policies is that society should never act in ways that express support for the practice in question, which in line with the minority of respondents' comments. However, this argument is questionable. A more pragmatic approach would also allow for follow-ups and evaluation of virginity certificates and hymen restorations, which has been demonstrated in Holland. Hence, there are some obvious advantages with this pragmatic approach compared with the restrictive one espoused by a large minority of Swedish physicians and Swedish policy makers in this area.

Acknowledgements Financial support from the Swedish Crime Victim Compensation and Support Authority is gratefully acknowledged.

Contributors Both the authors contributed equally to developing the argument of the manuscript, drafting and revising the manuscript, and read and approved the final manuscript.

Funding The Swedish Crime Victim Compensation and Support Authority. Competing interests None.

Ethics approval The regional research ethics committee at Karolinska Institutet, Stockholm, Sweden.

Provenance and peer review Not commissioned; externally peer reviewed.

\section{REFERENCES}

1 UNFPA: State of world population 2000. Chapter 3: "Honour" Killings. Ending Violence against Women and Girls. A Human Rights and Health Priority. http://www.unfpa.org/swp/2000/english/index.html

2 Tännsjö T. Hedersmoral—ett problem i västerländsk sjukvård? (in Swedish: Morality of honour-a problem in Western healthcare?). Tidskrift för politisk filosofi 2011;1:6-26.
3 Juth N, Hansson SO, Tännsjö T, et al. Honour related threats and human rights: a qualitative study of Swedish healthcare staffs' attitudes towards young women requesting virginity certificates or hymen surgery. Eur I Contracept Reprod Health Care 2013:18:451-9.

4 van Moorst BR, van Lunsen RH, van Dijken DK, et al. Backgrounds of women applying for hymen reconstruction, the effects of counselling on myths and misunderstandings about virginity, and the results of hymen reconstruction. Eur J Contracept Reprod Health Care 2012:17:93-105.

5 Cook RJ, Dickens BM. Hymen reconstruction: Ethical and legal issues. Int I Gynecol Obstet 2009;107:266-9.

6 Ahmadi A. Ethical issues in hymenoplasty: views from Tehran's physicians. J Med Ethics 2014;40:429-30.

7 Essén B, Blomkvist A, Helström L, et al. The experience and responses of Swedish health professionals to patients requesting virginity restoration. Reprod Health Matters 2010;18:38-46.

8 Sabuni N, Heimer G. Läkare ska inte göra "oskuldsoperationer". Svenska Dagbladet, 23 Nov 2011. http://www.svd.se/opinion/brannpunkt/lakare-skainte-gora-oskuldsoperationer_6653602.svd (accessed 25 Jun 2013).

9 Nationellt Centrum för Kvinnofrid (in Swedish: National Centre for Knowledge on Men's Violence Against Women). Att möta patienter som söker för oro kring oskuld och heder. (To encounter patients who are concerned about virginity and honour). http://kunskapsbanken.nck.uu.se/nckkb/nck/publik/fil/visa/296/NCK-rapport_2011-2_ Att_mota_patienter_www.pdf (accessed 25 Jun 2013).

10 Amy JJ. Certificates of virginity and reconstruction of the hymen. Eur J Contracept Reprod Health Care 2008;13:11-3.

11 Villarreal H, Fogg C. Syringe-exchange programs and HIV prevention: if they're effective, what's the controversy? Am I Nurs 2006;106:58-63.

12 Kerr T, Small W, Buchner C, et al. Syringe sharing and HIV incidence among injection drug users and increased access to sterile syringes. Am J Public Health 2010;10:1449-53.

13 Small D, Drucker E. Return to Galileo? The Inquisition of the International Narcotic Control Board. Harm Reduct J 2008;5:16.

14 Wilkinson S. Choosing Tomorrow's Children: The Ethics of Selective Reproduction. Oxford: Clarendon Press, 2010:148-85.

15 Lynöe N, Mattsson B. Doctor for patients or doctor for society? Comparative study of GPs' and psychiatrists' assessment of clinical practice. Scand I Prim Health Care 2004:22:228-32.

16 Helgesson $\mathrm{G}$, Lynöe N. Should physician fake diagnoses to help their patients? J Med Ethics 2008:34:133-36. 